\title{
Thomas Gehring
}

\section{Internationale Institutionen, Global Governance und Steuerungsfragen}

Das Gebiet der internationalen Institutionen, des Regierens jenseits des Nationalstaates sowie seiner eher präskriptiven Variante der Global Governance gehört seit nunmehr 15 Jahren zu den besonders wichtigen und dynamischen Bereichen der deutschsprachigen Internationalen Beziehungen. So stellen Volker Rittberger und Hartwig Hummel (1990: 34) und Michael Zürn (2003: 24) in ihren einleitenden Beiträgen zu den jeweiligen »state of the art«-Bänden der Internationalen Beziehungen in Deutschland gleichermaßen fest, dass der Neo-Institutionalismus zu einem gemeinsamen Fundament der Forschung geworden ist. Auf diese Hinwendung zu einer theorieorientierten und - in einem weiteren Sinne - institutionenbezogenen IB-Forschung in Deutschland lässt sich auch die Gründung der Zeitschrift für Internationale Beziehungen (ZIB) zurückführen. Es ist deshalb nicht erstaunlich, dass der hier zu behandelnde Sachbereich den wahrscheinlich größten und wichtigsten Einzelbereich der Veröffentlichungstätigkeit in der ZIB bildet.

In diesem Beitrag will ich - den Vorgaben der Herausgeber entsprechend zunächst ausleuchten, inwieweit die ZIB ihrem eigenen Anspruch auf diesem Sachgebiet gerecht geworden ist und wo dies noch nicht vollständig gelungen ist (Abschnitt 1) und anschließend auf zwei Probleme hinweisen, denen sich die ZIB meiner Meinung nach wird stellen müssen. Eines dieser Probleme, nämlich die Frage der thematischen und methodischen Ausrichtung in der Zukunft, ist sachgebietsspezifisch (Abschnitt 2), während das andere, die Frage der Verortung der ZIB in der internationalen Landschaft der theoretisch orientierten IB-bezogenen Zeitschriften, naturgemäß sachgebietsübergreifend ist (Abschnitt 3).

\section{Inwieweit hat die ZIB die von ihr selbst gesetzten Ziele erreicht?}

Es besteht wohl kein Zweifel daran, dass die ZIB für den hier behandelten Sachbereich ihre wesentlichen Ziele weitestgehend eingelöst hat - wahrscheinlich stärker als dies für die Gebiete gilt, die in den anderen Beiträgen behandelt werden. Sie ist nicht zuletzt aufgrund ihres rigorosen anonymisierten Begutachtungsverfahrens - zu einer qualitativ hochwertigen Zeitschrift geworden, an der sich Forschung und Lehre in Deutschland orientieren. Autoren finden im deutschsprachigen Raum kein ebenbürtiges Publikationsorgan, da die Politische Vierteljahresschrift einen wesentlich breiter gesteckten Themenbereich abdecken muss und andere Zeitschriften, etwa Internationale Politik und die Blätter für deutsche und internationale Politik, anwendungsnäher und deshalb - gewolltermaßen - weniger grundlagenbezogen 
sind. Für Leser gibt es deshalb kein besseres Forum, um sich über die deutschsprachige theoriegeleitete und institutionenbezogene Forschung zu informieren. Insofern hat die ZIB gerade in diesem Sachbereich ohne Frage zu einer starken Identitätsbildung beigetragen. Allerdings kann der Erfolg auch selbst zum Problem werden (vgl. Abschnitt 2).

Wenn man genauer hinsieht, werden naturgemäß Nuancierungen erkennbar. Das Feld selbst ist unklar umgrenzt. So stellt die ZIB-Debatte um die Bedeutung von Kommunikation und Argumentation in den Internationalen Beziehungen zunächst einmal eine Auseinandersetzung mit den IB-Theorien dar, hat aber - vor allem in der operationalisierten Form der Kategorien von Arguing und Bargaining - auch unmittelbare Auswirkungen auf Verhandlungen, die wiederum eine wichtige Vorstufe für organisierte Kooperation sowie für den Aufbau und die Entwicklung internationaler Institutionen bilden. Und die Aktivitäten der NATO im Kosovo-Konflikt sind für die einen eine Frage von Krieg und Frieden, während andere sie als Politikergebnis einer internationalen Organisation betrachten.

Zürn (2003: 26) und Jachtenfuchs (2003) gliedern das Feld in drei Teilbereiche: Die kooperationstheoretische Analyse fragt nach den Bedingungen, unter denen Kooperation sowie die Steuerung durch internationale Institutionen im Schatten der »Anarchie« des internationalen Systems möglich ist und reflektiert damit die politics-Dimension. Die steuerungstheoretische Perspektive untersucht hingegen die Steuerungswirkungen und impliziert deshalb eine starke policy-Dimension. Die Diskussion um Global Governance wirft zusätzlich die Frage des Zusammenspiels verschiedener sektoraler Institutionen und unterschiedlicher Akteure sowie die Fragen von Legitimität und Gerechtigkeit auf. Damit kommt die polity-Dimension verstärkt ins Spiel. Man kann überdies zwischen positiver Analyse und normativer bzw. präskriptiver Ausrichtung unterscheiden.

Bei aller Vorsicht hinsichtlich der Trennschärfe dieser Felder und der Zuordnung einzelner Beiträge zeigt sich doch, dass nicht alle Teilbereiche der theoriegeleiteten und institutionenbezogenen Forschung gleichermaßen gut vertreten sind. Am weitaus stärksten besetzt ist die Zelle der im weiteren Sinn kooperationstheoretischen politics-Forschung, die sich mit Fragen der Entstehungsbedingungen von Kooperation und einzelnen internationalen Institutionen befasst, deren Entwicklung sowie dem Einfluss bestimmter Akteure darauf. In der Anfangszeit der Zeitschrift gehörten dazu Beiträge zur Bedeutung von Zweiebenenspielen für den Aufbau internationaler Regime (Zangl 1994), zur Erweiterung der NATO aus der Clubgut-Perspektive (Bernauer 1995) und zur Bankenregulierung (Lütz 1999; Genschel/Plümper 1996). In der jüngeren Zeit ging es etwa um das strategische Signalisieren bei internationalen Verhandlungen (Thurner et al. 2003) und um Erklärungen für Regime, für die es aus einer rein funktionalistischen Logik heraus eigentlich keinen Bedarf gibt (Daase 2003a, 2003b). Daneben gewinnen neue, »konstruktivistische« Einflussfaktoren, etwa NGOs und der Interaktionsmodus des Arguing, an Bedeutung. Damit wird auch die Staatszentriertheit der klassischen Regimeforschung durchbrochen. Dies alles scheint dem Befund von Klaus Dieter Wolf und Gunther Hellmann (2003: 597) zunächst zu widersprechen, dass die deutsche IB-Forschung sich nur 
noch innovativen Themen zuwende und dabei die klassischen Fragen der internationalen Politik vernachlässige. Allerdings nimmt die Besetzung dieser Zelle im Zeitverlauf deutlich ab.

Etwa zeitgleich mit der Abnahme der institutionenbezogenen Kooperationsforschung gewinnen seit der Jahrhundertwende weiterreichende Fragen der Global Governance stark an Bedeutung. Sie zeichnen sich einerseits durch eine breitere, nicht auf einzelne Institutionen gerichtete Perspektive und vielfach auch durch einen normativen Ansatz aus. Eingeleitet wurde diese Entwicklung durch das Themenheft »Globalisierung und die Handlungsfähigkeit der Nationalstaaten « (ZIB 2/2000), dessen Beiträge durchweg analytischen Charakter haben. Dies gilt auch für die Untersuchung der Durchsetzung von Menschenrechten durch die Interaktion nationaler, transnationaler und staatlicher Akteure. Im Zuge der Global Governance-Forschung tritt jedoch eine verstärkt normative Forschung zutage. So werden Fragen der demokratischen Legitimation globaler Politiknetzwerke (Dingwerth 2003), der transnationalen deliberativen Demokratie (Schmalz-Bruns 1999) und der symbolischen Integration der internationalen Gemeinschaft durch die Menschenrechte (Bonacker/Brodocz 2001) diskutiert.

Mäßig besetzt, allerdings mit leicht zunehmender Tendenz, ist schließlich das Feld der policy-orientierten Steuerungsperspektive. So wird, normativ, der KosovoKrieg der NATO im Lichte von Theorien des gerechten Krieges (Mayer 1999) bewertet oder, präskriptiv, der Aufbau einer Weltumweltorganisation gefordert oder abgelehnt (Biermann/Simonis 2000; Gehring/Oberthür 2000) und über den hegemonialen Diskurs über Global Governance diskutiert (Brandt 2003; Weller 2003). Es werden die Erfolgsaussichten dicht oder schlank institutionalisierter Institutionen der regionalen Integration ausgelotet (Rüland 2002) und die Ursachen der abnehmenden Steuerungswirkung des Nichtverbreitungsregimes für Atomwaffen intensiv diskutiert (Daase 2003a, 2003b; Dembinski/Müller 2003; Wolf 2003).

Auffällig ist die zahlenmäßig untergeordnete Bedeutung solcher Beiträge, die nationale Ursachen für Aspekte der institutionenbezogenen internationalen Politik (second image) oder nationale Wirkungen internationaler Steuerungsbemühungen (second image reversed) untersuchen. Für die erste Perspektive finden sich lediglich zwei Beiträge zur (inter)nationalen Klimapolitik in Deutschland und den USA (Ulbert 1997) und über Zwei-Ebenen-Spiele (Zangl 1994); für die zweite, abgesehen von dem Themenheft zur Globalisierung und der Handlungsfähigkeit der Nationalstaaten, zwei Beiträge über die Durchsetzung von Menschenrechten und die Politik der Vereinten Nationen zur Gewalt gegen Frauen (Joachim 2001; Forschungsgruppe Menschenrechte 1998). Insofern ist die analytische Überschreitung der Grenze zwischen der nationalen und der internationalen Politik in der Publikationstätigkeit noch nicht so weit fortgeschritten, wie dies oft angenommen wird.

Was die behandelten Politikfelder angeht, so ist das Bild in Bezug auf die hier betrachteten institutionenbezogenen Beiträge ausgewogener als gelegentlich vermutet. Wolf/Hellmann (2003: 597) äußern in ihrem Beitrag zur Zukunft der deutschen IB-Forschung die Besorgnis, dass nur noch Fragen der Zivilgesellschaft und der Umweltpolitik Interesse finden und Fragen von Krieg und Frieden zunehmend ver- 
nachlässigt werden könnten. In den einschlägigen Beiträgen der ZIB kommen Menschenrechte und Umweltpolitik etwa gleich oft, d. h. sechs bis siebenmal vor. Dagegen werden institutionenbezogene Sicherheitsfragen in über zehn Beiträgen behandelt. Selbst wenn man die Forumsbeiträge zu der bereits erwähnten Diskussion um die Bedeutung des Nichtverbreitungsregimes für Atomwaffen nicht mitzählt, sind institutionenbezogene Sicherheitsfragen keineswegs unterrepräsentiert. Lediglich Fragen mit starkem wirtschaftlichen Bezug sind etwas weniger vertreten, aber behandelt werden die internationale Bankenregulierung, der Steuerwettbewerb zwischen Staaten und die regionale Wirtschaftsintegration außerhalb der EU.

Insgesamt lässt sich feststellen, dass es der ZIB gelungen ist, hochwertige Beiträge $\mathrm{zu}$ einer breiten Palette theoriegeleiteter und institutionenbezogener Forschungsfragen zu gewinnen und damit einen großen Teil der relevanten deutschen Institutionen- und Governance-Forschung abzubilden. Dabei hat die ZIB - nolens volens - den Trend der deutschen IB-Forschung von der enger analytisch ausgerichteten Institutionenforschung zur breiter angelegten und stärker normativen Governance-Forschung mit vollzogen.

\section{Das Problem der thematischen und theoretischen Ausrichtung}

Ist also alles in Ordnung? Die erfolgreiche Etablierung der ZIB als Identifikationspunkt für die deutschsprachige Institutionen- und Governance-Forschung weist der Zeitschrift eine disziplindefinierende Rolle zu. Wenn AutorInnen die ZIB in dieser Rolle wahrnehmen, werden sie sich an diesen Maßstäben orientieren, um selbst Beiträge erstellen zu können, die von anderen als qualitativ hochwertig angesehen werden. Und AutorInnen der ZIB sorgen als Gutachter dafür, dass nur solche Beiträge in der Zeitschrift veröffentlicht werden, die diesen Maßstäben genügen (Hellmann/ Müller 2003: 381-385). Es entsteht eine selbstreferenzielle Beziehung, die der deutschen IB-Forschung so lange förderlich ist, wie sie dazu beiträgt, ihre Qualität zu steigern. Dies ist durch das für die Disziplin zunächst neue doppelt anonyme Begutachtungsverfahren zweifellos gelungen. Allerdings ist damit auch die Gefahr von Fehlentwicklungen verbunden: Wenn die ZIB »falsche« Maßstäbe setzt, wird ein Teil der deutschen Institutionen- und Governance-Forschung unweigerlich in eine falsche Richtung gelenkt.

Das Problem der ZIB liegt, jedenfalls was den hier behandelten Sachbereich betrifft, nicht so sehr in den stark theoretisch ausgerichteten Beiträgen. Die beiden derzeitigen geschäftsführenden Herausgeber haben zu Bedenken gegeben, dass die starke Theorieorientierung, die sich in Debatten niederschlage, womöglich nicht die Art von Wissen produziere, die die Gesellschaft benötige (Hellmann/Müller 2003: 381f). Dies könne man beispielsweise dadurch beheben, dass Praktiker verstärkt in den Begutachtungsprozess einbezogen würden. Das scheint mir aus zwei Gründen nicht angebracht zu sein: Zum einen verzichten nur wenige Beiträge, die auf Institutionen und Governance-Fragen bezogen sind, auf einen empirischen Bezug; zum anderen sollte die Redaktionspolitik nicht an der Forderung nach einem unmittelba- 
ren Anwendungsbezug ausgerichtet werden, wenn die Zeitschrift den Identifikationspunkt für die theoriegeleitete IB-Forschung bilden soll.

Allerdings leidet die ZIB unter einem Mangel an Beiträgen, in deren Zentrum die theoriegeleitete Klärung empirischer Fragen steht. Nach meinen Eindruck hat die Zeitschrift International Organization, die für den hier behandelten Sachbereich sicher als Vorbild gelten kann, einen weitaus stärkeren Empiriebezug als die ZIB. Obwohl die Institutionen- und Governance-Forschung per se empirische Zusammenhänge zum Gegenstand hat, werden in zahllosen Artikeln Analysekonzepte dargelegt, so dass die vorhandene Empirie nur den Stellenwert einer Illustration oder eines Beispiels einnimmt. Der Leser erhält dann in erster Linie Vorschläge darüber, wie er selbst einen bestimmten Ausschnitt »der Welt draußen« (auch) analysieren könnte oder welche normativen Gesichtspunkte er dabei beachten sollte, aber er erfährt nichts über diese Welt selbst. Demgegenüber sind empirisch orientierte Beiträge unterrepräsentiert, deren Schwerpunkt auf der Untersuchung interessanter empirischer Zusammenhänge liegt. Dies könnte etwa die Funktionsweise oder die Wirkungen einzelner wichtiger oder innovativer Institutionen (z. B. von Internationalem Währungsfond IWF, Weltbank oder der Weltstaudammkommission; vgl. aber Dingwerth 2003), die vergleichende Analyse der Steuerungswirkungen mehrerer Institutionen, die Wechselwirkung zwischen Institutionen oder die Folgen der Öffnung bestimmter Institutionen für Nichtregierungsorganisationen betreffen. Natürlich darf eine stärkere Empirieorientierung nicht zur Publikation zahlloser isolierter Fallstudien führen. Insofern ist ein ausgeprägter Theoriebezug für jeden Beitrag unverzichtbar. Er dient dann jedoch nicht in erster Linie der Diskussion theoretischer Aspekte, sondern als Instrumentarium für die sinnvolle Strukturierung der empirischen Forschung innerhalb eines breiteren, auf kumulative Erkenntnis angelegten Forschungsprogramms.

Ein erster Schritt zur verstärkten Einwerbung theoriegeleiteter, aber empirisch orientierter Beiträge könnte in einem entsprechenden Aufruf zum Einreichen empirisch ausgerichteter Beiträge im Editorial bestehen. Bei der Durchsicht der von den jeweiligen geschäftsführenden Herausgebern verfassten Editorials fällt auf, dass das Interesse am Allgemeinen bzw. Verallgemeinerbaren stark betont wird (Wolf 1994), während explizite Ermunterungen zur Publikation empirisch orientierter Artikel fehlen. Wenn dies insbesondere bei den nach Orientierung suchenden NachwuchsforscherInnen zu dem gelegentlich zu vernehmenden Einruck führt, die ZIB nähme nur Beiträge mit theoretischem Schwerpunkt an, besteht die ernste Gefahr einer signifikanten Fehlentwicklung der Disziplin. Überdies würde die ZIB durch die vermehrte Publikation dezidiert empiriebezogener Beiträge gewissermaßen als Nebeneffekt automatisch auch für Außenstehende interessanter.

\section{Das Problem der Verortung der ZIB in der Landschaft der internationalen Fachzeitschriften}

Ein zweites Problem besteht in der Verortung der ZIB in der internationalen Landschaft der IB-bezogenen und theoretisch orientierten Zeitschriften. In Bezug auf die 
Institutionen- und Governance-Forschung fällt auf, dass die Ergebnisse einiger der auf diesem Gebiet tätigen deutschen Forscher, die in internationalen Fachzeitschriften publizieren, kaum oder gar nicht in der ZIB erscheinen. Mit der zunehmenden Konkurrenzfähigkeit und Internationalisierung der deutschsprachigen IB-Forschung wird sich die Konkurrenz der ZIB zu hochrangigen internationalen Zeitschriften (z. B. dem European Journal of International Relations, International Organization etc.) unweigerlich verstärken. Gerade international besonders wettbewerbsfähige Beiträge werden dann aufgrund des höheren Renommees internationaler Publikationen für die ZIB zunehmend unerreichbar. Überdies liegt es nahe, Beiträge allein aufgrund der Sprache auch dann nicht bei der ZIB, sondern bei zweit- oder drittrangigen englischsprachigen Zeitschriften einzureichen, wenn sie bei den am höchsten renommierten Organen scheitern oder für internationale Konferenzen in englischer Sprache verfasst worden sind.

Meines Erachtens muss sich die ZIB diesem Problem offener stellen als bisher. Solange sie nicht in Konkurrenz beispielsweise zu dem European Journal of International Relations treten, sondern den Identifikationspunkt für die deutschsprachige IB-Forschung bilden will, kann ihr nicht daran gelegen sein, Autoren vor die Wahl $\mathrm{zu}$ stellen, entweder in Deutschland oder international zu veröffentlichen. Diese Wettbewerbssituation wäre auch für die Disziplin insgesamt nicht unproblematisch. Wenn Autoren sich entscheiden müssten, ob sie wettbewerbsfähige Ergebnisse national oder international veröffentlichen wollen, würde jede ZIB-Publikation den Einfluss der deutschsprachigen IB-Forschung auf die internationale Diskussion automatisch reduzieren. Deshalb müsste die ZIB bestrebt sein, Ergebnisse möglichst jedes guten deutschsprachigen Forschungsprojektes unabhängig davon zu veröffentlichen, ob sie (auch) international publiziert werden oder worden sind, soweit dies urheberrechtlich zulässig ist. Gleichzeitig sollte die Redaktion darüber nachdenken, ob die ZIB, trotz ihres aus guten Gründen im wesentlichen deutschsprachigen Charakters, in Zukunft auch in englischer Sprache verfasste Beiträge anzunehmen bereit ist. Aus eigener Erfahrung weiß ich, dass thematisch passende Manuskripte gelegentlich nicht eingereicht werden, weil die Zeit oder Lust für die Rückübersetzung ins Deutsche fehlen. In jedem Fall gilt es auch in dieser Hinsicht, Publikationsmöglichkeiten (und deren Grenzen) zum Beispiel im Rahmen des Editorials gegenüber potenziellen Autoren regelmäßig so deutlich zu machen, dass die Zahl der eingereichten Manuskripte nicht durch ungewollte Selbstbeschränkung unnötig reduziert wird. 


\section{Literatur}

Bernauer, Thomas 1995: Theorie der Klub-Güter und die Osterweiterung der NATO, in: Zeitschrift für Internationale Beziehungen 2: 1, 79-106.

Biermann, Frank/Simonis, Udo E. 2000: Institutionelle Reform der Weltumweltpolitik? Zur politischen Debatte um die Gründung einer »Weltumweltorganisation«, in: Zeitschrift für Internationale Beziehungen 7: 1, 163-183.

Bonacker, Thorsten/Brodocz, André 2001: Im Namen der Menschenrechte. Zur symbolischen Integration der internationalen Gemeinschaft durch Normen, in: Zeitschrift für Internationale Beziehungen 8: 2, 179-208.

Brand, Ulrich 2003: Nach der Krise des Fordismus. Global Governance als möglicher hegemonialer Diskurs des internationalen Politischen, in: Zeitschrift für Internationale Beziehungen 10: 1, 143-166.

Daase, Christopher 2003a: Der Anfang vom Ende des nuklearen Tabus. Zur Legitimitätskrise der Weltnuklearordnung, in: Zeitschrift für Internationale Beziehungen 10: 1, 7-41.

Daase, Christopher 2003b: Nonproliferation und das Studium internationaler Legitimität. Eine Antwort auf meine Kritiker, in: Zeitschrift für Internationale Beziehungen 10: 2, 351-364.

Dembinski, Matthias/Müller, Harald 2003: Mehr Ratio als Charisma: Zur Entwicklung des nuklearen Nichtverbreitungs-Regimes vor und nach 1995. Eine Replik auf Christopher Daase, in: Zeitschrift für Internationale Beziehungen 10: 2, 333-350.

Dingwerth, Klaus 2003: Globale Politiknetzwerke und ihre demokratische Legitimation. Eine Analyse der Weltstaudammkommission, in: Zeitschrift für Internationale Beziehungen 10: 1, 69-110.

Forschungsgruppe Menschenrechte 1998: Internationale Menschenrechtsnormen, transnationale Netzwerke und politischer Wandel in den Ländern des Südens, in: Zeitschrift für Internationale Beziehungen 5: 1, 5-42.

Gehring, Thomas/Oberthür, Sebastian 2000: Was bringt eine Weltumweltorganisation? Kooperationstheoretische Anmerkungen zur institutionellen Neuordnung der internationalen Umweltpolitik, in: Zeitschrift für Internationale Beziehungen 7: 1, 185-211.

Genschel, Philipp/Plümper, Thomas 1996: Wenn Reden Silber und Handeln Gold ist. Kooperation und Kommunikation in der internationalen Bankenregulierung, in: Zeitschrift für Internationale Beziehungen 3: 2, 225-254.

Hellmann, Gunther/Müller, Harald 2003: Editing (I)nternational (R)elations: A Changing World, in: Journal of International Relations and Development 6: 4, 372-389.

Jachtenfuchs, Markus 2003: Regieren jenseits der Staatlichkeit, in: Hellmann, Gunther/Wolf, Klaus Dieter/Zürn, Michael (Hrsg.): Die neuen Internationalen Beziehungen. Forschungsstand und Perspektiven in Deutschland, Baden-Baden, 495-518.

Joachim, Jutta M. 2001: NGOs, die Vereinten Nationen und Gewalt gegen Frauen: AgendaSetting, Framing, Gelegenheits- und Mobilisierungsstrategien, in: Zeitschrift für Internationale Beziehungen 8: 2, 209-242.

Lütz, Susanne 1999: Zwischen »Regime« und »kooperativem Staat«. Bankenregulierung im internationalen Mehrebenensystem, in: Zeitschrift für Internationale Beziehungen 6: 1, 9-40.

Mayer, Peter 1999: War der Krieg der NATO gegen Jugoslawien moralisch gerechtfertigt? Die Operation »Allied Force« im Lichte der Lehre vom gerechten Krieg, in: Zeitschrift für Internationale Beziehungen 6: 2, 287-322.

Schmalz-Bruns, Rainer 1999: Deliberativer Supranationalismus. Demokratisches Regieren jenseits des Nationalstaats, in: Zeitschrift für Internationale Beziehungen 6: 2, 185-244.

Rittberger, Volker/Hummel, Hartwich 1990: Die Disziplin »internationale Beziehungen« im deutschsprachigen Raum auf der Suche nach ihrer Identität. Entwicklung und Perspektiven, in: Rittberger, Volker (Hrsg.): Theorien der Internationalen Beziehungen. Bestandsaufnahme und Forschungsperspektiven (PVS-Sonderheft 21), Opladen, 17-47.

Rüland, Jürgen 2002: »Dichte« oder »schlanke« Institutionalisierung? Der Neue Regionalismus im Zeichen von Globalisierung und Asienkrise, in: Zeitschrift für Internationale Beziehungen 9: 2, 175-208. 
Thurner, Paul W./Kroneberg, Clemens/Stoiber, Michael 2003: Strategisches Signalisieren bei internationalen Verhandlungen. Eine quantitative Analyse am Beispiel der Regierungskonferenz 1996, in: Zeitschrift für Internationale Beziehungen 10: 2, 287-320.

Ulbert, Cornelia 1997: Ideen, Institutionen und Kultur. Die Konstruktion (inter-)nationaler Klimapolitik in der BRD und in den USA, in: Zeitschrift für Internationale Beziehungen 4: 1, 9-40.

Weller, Christoph 2003: Die Welt, der Diskurs und Global Governance. Zur Konstruktion eines hegemonialen Diskurses - eine Replik auf Ulrich Brand, in: Zeitschrift für Internationale Beziehungen 10: 2, 365-382.

Wolf, Klaus Dieter 1994: Editorial, in: Zeitschrift für Internationale Beziehungen 1: 1, 3-13.

Wolf, Klaus Dieter/Hellmann, Gunther 2003: Die Zukunft der Internationalen Beziehungen in Deutschland, in: Hellmann, Gunther/Wolf, Klaus Dieter/Zürn, Michael (Hrsg.): Die neuen Internationalen Beziehungen. Forschungsstand und Perspektiven in Deutschland, Baden-Baden, 577-603.

Wolf, Reinhard 2003: Tabu, Verrechtlichung und die Politik der nuklearen Nichtverbreitung. Eine interessante Hypothese auf der Suche nach einem tatsächlichen Problem, in: Zeitschrift für Internationale Beziehungen 10: 2, 321-331.

Zangl, Bernhard 1994: Politik auf zwei Ebenen. Hypothesen zur Bildung internationaler Regime, in: Zeitschrift für Internationale Beziehungen 1: 2, 279-312.

Zürn, Michael 2003: Die Entwicklung der Internationalen Beziehungen im deutschsprachigen Raum seit 1989, in: Hellmann, Gunther/Wolf, Klaus Dieter/Zürn, Michael (Hrsg.): Die neuen Internationalen Beziehungen. Forschungsstand und Perspektiven in Deutschland, Baden-Baden, 21-46. 